\title{
Patient perception of pain care in hospitals in the United States
}

This article was published in the following Dove Press journal:

Journal of Pain Research

12 November 2009

Number of times this article has been viewed

\section{Anita Gupta' \\ Sarah Daigle ${ }^{2}$ \\ Jeffrey Mojica ${ }^{3}$ \\ Robert W Hurley ${ }^{4}$}

'Pain Management Division, Department of Anesthesiology, University of Pennsylvania School of Medicine, Philadelphia, PA, USA; ${ }^{2}$ Department of Anesthesiology and Critical Care, ${ }^{3}$ Department of Anesthesiology and Critical Care, Division of Pain Medicine, University of Pennsylvania, Philadelphia, PA, USA; ${ }^{4}$ Medical Director of the Johns Hopkins Pain Treatment Center, Division of Pain Medicine, Deparment of Anesthesiology and Critical Care Medicine, Johns Hopkins School of Medicine, Baltimore, MD, USA
Correspondence: Anita Gupta Assistant Professor, Pain Management Division, Department of Anesthesiology and Critical Care, University of Pennsylvania School of Medicine, Tuttleman Center, 1840 South Street, Second Floor, Philadelphia, PA 19146, USA Tel +l 2158937246

Email anita.gupta@uphs.upenn.edu
Study objective: Assessment of patients' perception of pain control in hospitals in the United States.

Background: Limited data are available regarding the quality of pain care in the hospitalized patient. This is particularly valid for data that allow for comparison of pain outcomes from one hospital to another. Such data are critical for numerous reasons, including allowing patients and policy-makers to make data-driven decisions, and to guide hospitals in their efforts to improve pain care. The Hospital Quality Alliance was recently created by federal policy makers and private organizations in conjunction with the Centers for Medicare and Medicare Services to conduct patient surveys to evaluate their experience including pain control during their hospitalization. Methods: In March 2008, the results of the Hospital Consumer Assessment of Healthcare Providers and Systems (HCAHPS) survey was released for review for health care providers and researchers. This survey includes a battery of questions for patients upon discharge from the hospital including pain-related questions and patient satisfaction that provide valuable data regarding pain care nationwide. This study will review the results from the pain questions from this available data set and evaluate the performance of these hospitals in pain care in relationship to patient satisfaction. Furthermore, this analysis will be providing valuable information on how hospital size, geographic location and practice setting may play a role in pain care in US hospitals.

Results: The data indicates that $63 \%$ of patients gave a high rating of global satisfaction for their care, and that an additional $26 \%$ of patients felt that they had a moderate level of global satisfaction with the global quality of their care. When correlated to satisfaction with pain control, the relationship with global satisfaction and "always" receiving good pain control was highly correlated $(r>0.84)$. In respect to the other HCAHPS components, we found that the patient and health care staff relationship with the patient is also highly correlated with pain relief $(r>0.85)$. The patients' reported level of pain relief was significantly different based upon hospital ownership, with government owned hospitals receiving the highest pain relief, followed by nonprofit hospitals, and lastly proprietary hospitals. Hospital care acuity also had an impact on the patient's perception of their pain care; patients cared for in acute care hospitals had lower levels of satisfaction than critical access hospitals.

Conclusions: The results of this study are a representation of the experiences of patients in US hospitals with regard to pain care specifically and the need for improved methods of treating and evaluating pain care. This study provides the evidence needed for hospitals to make pain care a priority in to achieve patient satisfaction throughout the duration of their hospitalization. Furthermore, future research should be developed to make strategies for institutions and policy-makers to improve and optimize patient satisfaction with pain care.

Keywords: pain care, HCAHPS, hospitals 


\section{Background}

In the last two decades, treatment of pain has become a major public health concern according to the Joint Commission on Accreditation of Healthcare Organizations (JCAHO). ${ }^{1}$ Much attention has been given to the quality of pain management of hospitalized patients with growing concern that poor pain control leads to avoidable chronic pain conditions. ${ }^{2,3}$ Major barriers to effective pain management have been identified including the inadequate knowledge of health care professionals, patients and the public; poor communication between care providers, poor communication between patients and care providers, lack of institutional commitment; regulatory concerns; and limited access to and reimbursement for interdisciplinary care. ${ }^{4}$ Even with strong efforts by multiple national organizations, including JCAHO, advances in our understanding of pain and the quality of pain management remains inconsistent at best.

Patient support groups, national medical policy makers, and private organizations have continued to emphasize the importance of health care reform and improved quality of care for patients throughout the United States. In order to accomplish this the Hospital Quality Alliance (HQA) was developed in coordination with private and government agencies to publicly report data on the quality of care in patients seen in US hospitals. ${ }^{5}$ The HQA in turn developed the Hospital Consumer Assessment of Healthcare Providers and Systems (HCAHPS) survey which is given to patients upon discharge from their hospital. A majority of US hospitals have cooperated with collecting and reporting this data to assist in improving patient care overall. In October 2008, Jha and colleagues evaluated the data in detail and identified a need for improvement in hospitals across the nation in a variety of areas including global patient satisfaction and pain care. ${ }^{6}$ To the best of our knowledge, no analysis of the HCAHPS data has been published regarding hospital performance on pain care.

Publicly evaluating data on clinical performance has catalyzed improvements in optimizing patient care in hospitals. ${ }^{7}$ Evaluating this data specifically focusing on the level of patient satisfaction with pain control will provide valuable insight into how specialists in pain medicine may be able to improve pain assessment and treatment. We addressed several questions in our review of this data: How do hospitals perform with respect to pain medicine care when compared to other hospitals in the US? How does global patient satisfaction relate to pain control? Do specific characteristics of hospitals including size and practice setting have a relationship to pain care? Does the type of hospital setting have a relationship with pain care?

\section{Methods}

\section{Survey design}

The Agency for Healthcare Research and Quality developed the HCAHPS survey which is comprised of 27 questions regarding their experience during their hospitalization and also demographic characteristics. The survey tool includes questions on pain control (Table 1):

During this hospital stay, how often was your pain well controlled? Always, usually, sometimes, or never.

During this hospital stay, how often did the hospital staff do everything they could to help you with your pain? Always, usually, sometimes, or never.

The survey tool assesses patient's global rating of the hospital care (Table 1):

Using any number from 0 to 10 , where 0 is the worst hospital possible and 10 is the best hospital possible, what number would you use to rate this hospital?

The global ratings were grouped by the Centers for Medicare and Medicaid Services (CMS) into one of three categories, 0-6 (minimal global satisfaction), 7-8 (moderate global satisfaction), or 9-10 (high global satisfaction) rather than made available individually. The details of the development of the survey, psychometric testing, and factor analyses used to create summary ratings within domains have been described previously. ${ }^{8-13}$ The CMS do not require participation in the HCAHPS survey tool. The data in this study are from the experiences of patients with respect to care delivered during the period from July 2006 through June 2007.

\section{Sampling and modes of survey administration}

Data included information from surveys from patients who spent at least one night in the hospital, were aged 18 years or older, were admitted with a nonpsychiatric diagnosis, and were alive at the time of discharge were eligible for the survey. The details of the inclusion and exclusion criteria are available from CMS at http://www.hcahpsonline.org/. All HCAHPS data available from CMS were adjusted for patient-level factors such as age, education, health status, and primary language. The preliminary work on HCAHPS data found that the adjustment for these variables helped reduce the nonresponse bias substantially. The complete details of the adjustment by mode, patient-factors, and for nonresponse, including specific models and adjustment coefficients are available from http://www.hcahpsonline.org/. 
Table I HCAHPS survey questions

\begin{tabular}{ll}
\hline Dimensions of care & Question \\
\hline Domain \#I & QI During the hospital stay, how often did nurses treat you with courtesy and respect? \\
Communication with nurses & Q2 During the hospital stay, how often did nurses listen carefully to you? \\
& Q3 During the hospital stay, how often did nurses explain things in a way you could \\
& understand?
\end{tabular}

Domain \#2

Communication with doctors

Domain \#3

Communication about medications

Domain \#4

Nursing services

Domain \#5

Discharge information

Domain \#6

Pain control

Domain \#7

Clean environment

Domain \#8

Quiet environment

Overall experience: Rating I to 10

Overall experience: Recommending hospital

About you
Q5 During the hospital stay, how often did doctors treat you with courtesy and respect?

Q6 During the hospital stay, how often did doctors listen carefully to you?

Q7 During the hospital stay, how often did doctors explain things in a way you could understand?

Q16 Before giving you any new medication, how often did hospital staff tell you what the medication was for?

QI7 Before giving you any new medication, how often did hospital staff describe possible side effects in a way you could understand?

Q4 During the hospital stay, after you pressed the call button, how often did you get help as soon as you wanted?

QII How often did you get help in getting to the bathroom or in using a bedpan as soon as you wanted?

Q18 After you left the hospital, did you go directly to your home, to someone else's home, or to another health facility?

Q19 During your hospital stay, did hospital staff talk with you about whether you would have the help you needed when you left the hospital?

Q20 During your hospital stay, did you get information in writing about what symptoms of health problems to look out for after you left the hospital?

Q13 During this hospital, how often was your pain well controlled?

QI4 During this hospital stay, how often did the hospital staff do everything they could to help you with your pain?

Q8 During the hospital stay, how often were your room and bathroom kept clean?

Q9 During your hospital stay, how often was the area around your room quiet at night?

Q21 Using any number from 0 to 10 , where 0 is the worst hospital possible and 10 is the best hospital possible, what number would you use to rate this hospital?

Q22 Would you recommend this hospital to your friends and family?

Q23 In general, how would you rate your overall health?

Q24 What is the highest grade or level of school that you have completed?

Q25 Are you of Hispanic or Latino origin or descent?

Q26 What is your race? Please choose one or more.

Q27 What language do you mainly speak at home?
Due to the various modes of survey administration and subsequently varying levels of response rates and response levels, mode-type adjustments are made to the data to ensure comparability across hospitals that use different survey administration modes. Regarding sample size and response rates: Hospitals reported results based on surveys of varying number of patients: 1,898 hospitals $(76 \%)$ had 300 or more completed responses, another 540 hospitals (21\%) had between 100 and 299 completed responses and 79 hospitals $(3 \%)$ reported data based on fewer than 100 completed responses. The response rates for surveys varied between hospitals with a mean response rate of $35.9 \%$. The data was adjusted to account for nonresponse bias. ${ }^{6}$ Complete details regarding the data set and survey tool have been described in detail by Jha and colleagues. ${ }^{6}$

\section{Statistical analysis}

We used multivariate logistic regression analysis to compare patient global satisfaction with patient level of pain control. 
Based upon previous statistical analysis by Jha and colleagues $^{6}$ of the survey we utilized the fraction of patients who rated the hospital in the highest category ( 9 or 10 on a scale of 0 to 10) as the foremost indication of high levels of patient satisfaction. We further assessed the national averages of hospital performance on pain control and correlated it to patient satisfaction.

\section{Results \\ Hospital characteristics}

The data comprised a total of 2,429 (60.2\%) hospitals out of a total of 4,032 hospitals that report any quality data to the HQA program. Of the reporting hospitals, greater than $75 \%$ of the hospitals had 300 or more patients who responded to the survey, whereas only $3 \%$ had fewer than 100 respondents. On average, $36 \%$ of the patients who were invited to participate chose to do so. Previous evaluation of the data demonstrates that hospitals that were large and private not-for-profit, hospitals with intensive care units, teaching hospitals, and hospitals located in urban areas and in the northeast US were more likely to report HCAHPS data than not to report the data and those that did not report HCAHPS data have been described in detail by Jha and colleagues. ${ }^{6}$

\section{Patients' satisfaction and relationship to pain control}

On average, $63 \%$ of patients gave their care a high global rating ( 9 or 10), and an additional $26 \%$ rated their care as 7 or 8 , whereas only $11 \%$ gave a rating of 6 or less (Figure 2 ). High global satisfaction of 9 to 10 ratings were positively associated with patients reporting high levels of pain control with a correlation coefficient of greater than 0.84 . Global satisfaction of $<6$ was negatively associated with patients reporting minimally controlled pain with a correlation coefficient of 0.84 . Figure 3 summarizes the correlation of well controlled pain with specific HCAHPS components. After a multivariate logistic regression analysis of the data, the data reveals also that patients who feel they have good communication with their doctors and/or nurses appear to be the greatest indicator of pain relief.

\section{Geographic relationship to pain control}

Patients in Alabama reportedly had the highest levels of pain relief, with an average of $77.4 \% \pm 9.734 \%$, whereas patients in Hawaii reported the lowest percentage of pain relief, with an average of $58.4 \% \pm 9.193 \%$ (Table 2). This represents a broad range of scores, with the highest states scoring $>70 \%$.

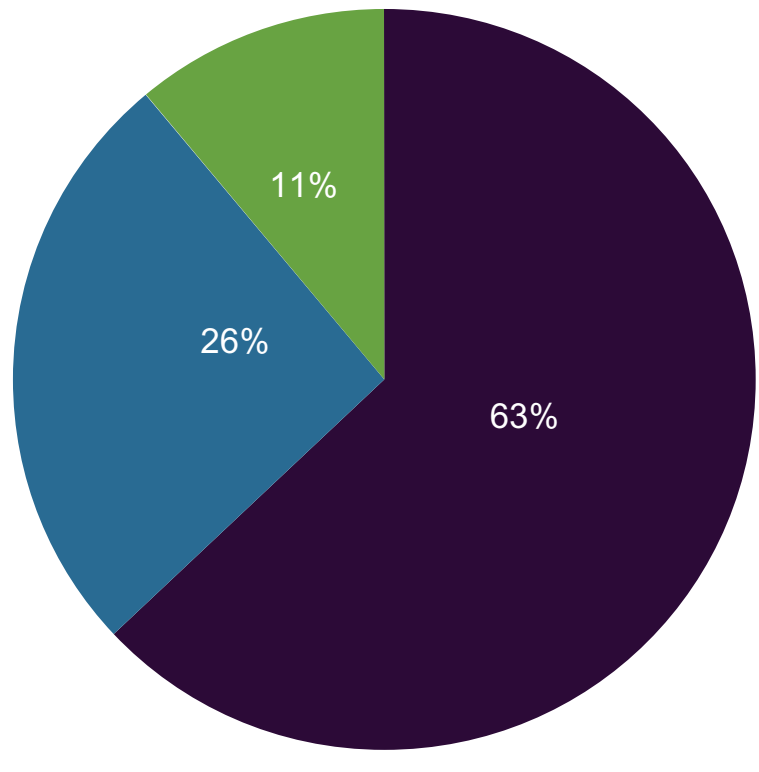

High Global Rating 9 or 10

7 or 8

\section{6 or less}

Figure I National percentage of patients reporting level of global satisfaction.

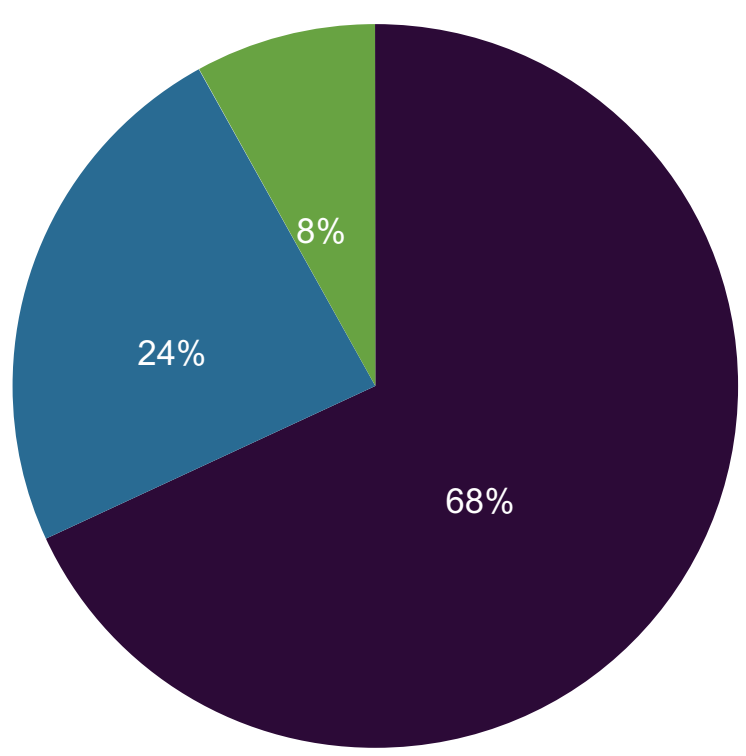

Pain always well controlled

Pain usually well controlled

Pain never well controlled

Figure 2 National percentage of patients reporting level of pain control. 


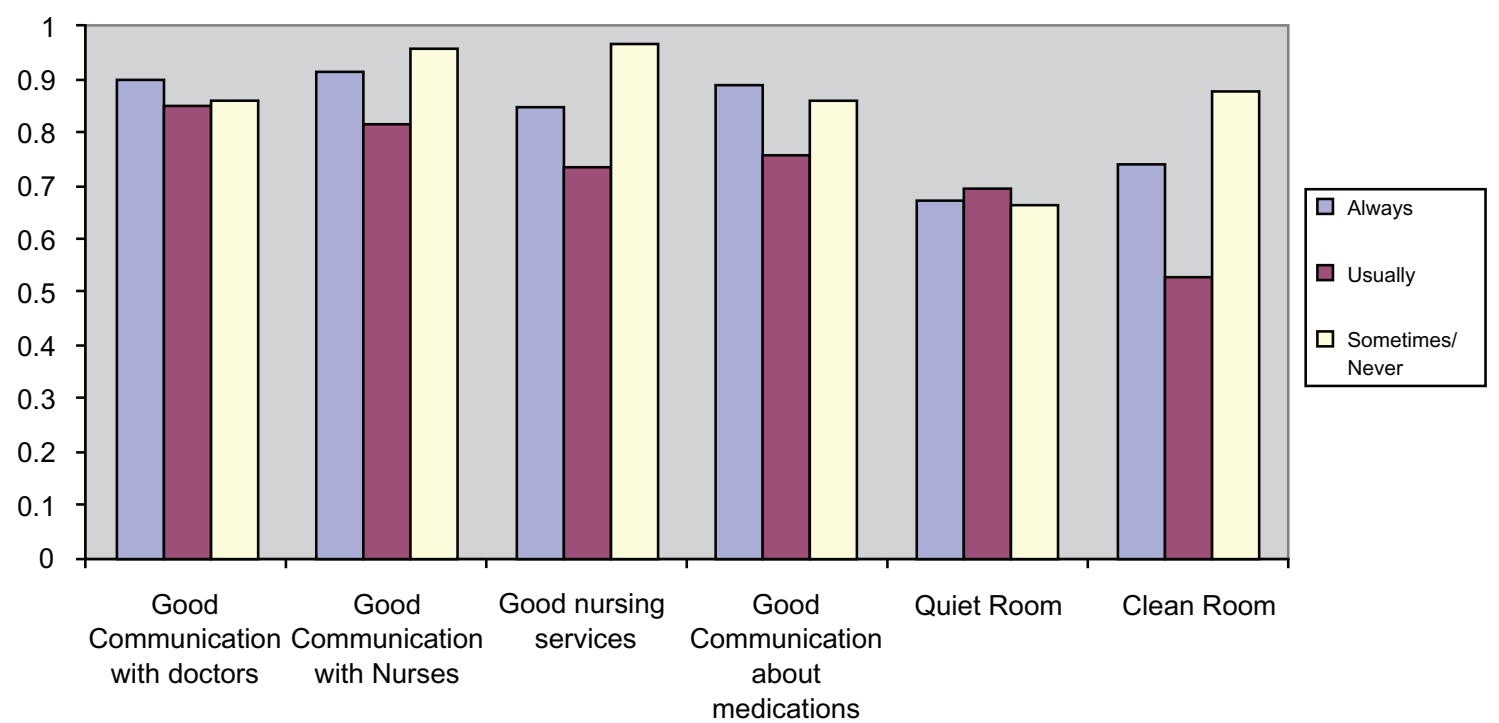

HCAHPS Component

Figure 3 Pearson coefficient of pain control and specific HCAHPS components.

Abbreviation: HCAHPS, Hospital Consumer Assessment of Healthcare Providers and Systems.

States that scored $<65 \%$ represent the states with the lowest levels of pain relief.

\section{Type of hospital relationship to pain control}

Additionally, the responding HCAHPS hospitals were divided into two categories: acute care hospitals (community care hospitals) and critical access hospitals (tertiary care hospitals). On average, approximately $71 \% \pm 33.36 \%$ of

Table 2 State performance on pain care based on HCAHPS survey

\begin{tabular}{ll}
\hline State & $\begin{array}{l}\text { Percentage of patients } \\
\text { with well controlled pain }\end{array}$ \\
\hline Top ranked & $77.4 \pm 9.734$ \\
I. Alabama & $71.6 \pm 6.843$ \\
2. Louisiana & $71.9 \pm 8.614$ \\
3. Oklahoma & $72.2 \pm 3.974$ \\
4. Maine & $71.4 \pm 3.693$ \\
5. New Hampshire & \\
Bottom ranked & $65.0 \pm 5.747$ \\
I. New York & $62.2 \pm 6.983$ \\
2. Florida & $63.3 \pm 2.081$ \\
3. District of Columbia & $59.1 \pm 7.594$ \\
4. Nevada & $58.4 \pm 9.193$ \\
5. Hawaii &
\end{tabular}

Note: Plus-minus values are means $\pm S D$.

Abbreviations: HCAHPS, Hospital Consumer Assessment of Healthcare Providers and Systems; SD, standard deviation. patients in critical access hospitals rated their pain as always well controlled, compared to only $67 \% \pm 7.307 \%$ of patients in acute care hospitals $(P<0.0001)$ (Table 3)

\section{Hospital ownership relationship to pain control}

The percentage of patients that reported well controlled pain were averaged and categorized based on hospital ownership (either government, nonprofit, or proprietary). We have shown that patients hospitalized in government-owned hospitals have the highest ratings of pain relief, followed by nonprofit hospitals, and then proprietary hospitals (Figure 4). On average, government hospitals report $69.4 \% \pm 7.295 \%$ of patients with well controlled pain upon discharge; nonprofit hospitals had report $67.8 \% \pm 5.416 \%$ of patients with well controlled pain upon discharge; and proprietary hospitals report $64.6 \% \pm 7.307 \%$ of patients with well controlled pain upon discharge. Furthermore, government-owned hospitals were noted to have a statistically significant difference compared with nonprofit hospitals and also with proprietary hospitals. Additionally nonprofit hospitals in comparison to proprietary hospitals also had a statistically significant difference $(P<0.05)$ (Figure 4).

\section{Discussion}

Inadequate pain control leads to delayed wound healing ${ }^{14}$ and is a risk factor for the development of chronic pain syndromes. ${ }^{2,15,16}$ Pain in the hospitalized patient has direct 
Table 3 Comparison of pain relief between critical care hospitals and critical access hospitals

\begin{tabular}{llll}
\hline Hospital type & $\mathbf{N}$ & Average & Standard deviation \\
\hline Acute care hospital & 2308 & 67.12 & 38.14 \\
Critical access hospital & 294 & 70.92 & 33.36 \\
\hline
\end{tabular}

Notes: Two tailed $t$-test $=9.99665, P \leq 0.0001$.

and indirect costs to the individual patient and society. In the acute hospital setting, unrelieved pain leads to longer hospital stays and higher mortality. ${ }^{17}$ Likewise, under treated chronic pain results in avoidable hospital admissions. Pain also will affect psychosocial factors which may cause an increased risk of depression, anxiety, substance abuse. ${ }^{18}$ Overall, inadequate pain care leads to poor patient outcomes and higher health care costs making this issue a public health problem.

The results of this study review the level of pain control overall delivered across the US. Sixty-eight percent of patients reported adequate pain control at a time when immense efforts by various organizations are in place to improve pain care. The HCAHPS survey has provided baseline data for national performance in pain care and emphasizes the need for reevaluation of the methods for providing pain care in hospitals. Furthermore, the results of this study elucidate the current relationship between quality of pain care and global patient satisfaction. This association will likely be even more convincing with higher hospital response rates should the HCAHPS survey be made mandatory by CMS for hospitals providing services to Medicare patients.

In today's rapidly progressing practice of medicine, pain care is in need for advancement. In January 2001, pain management standards were implemented by JCAHO to emphasize an interdisciplinary approach, individualized patient pain control plans, assessment (11-point scale), frequent reassessment of pain, use of pharmacologic and nonpharmacologic strategies, and establishment of a formalized approach. ${ }^{19}$ Although there has been improved appreciation and assessment of pain, the "fifth vital sign", the results

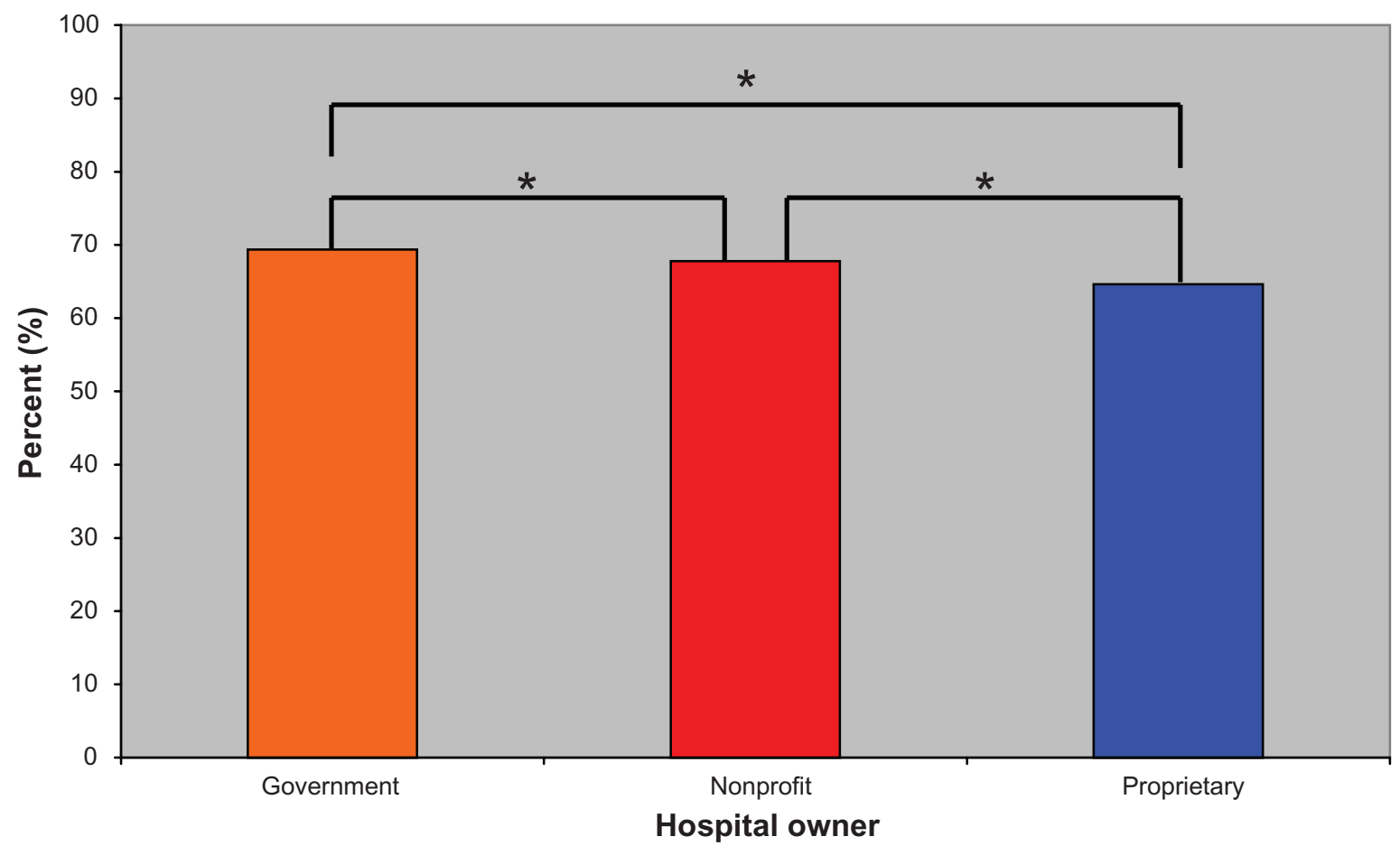

\begin{tabular}{|l|c|c|c|}
\hline \multicolumn{1}{|c|}{ Groups } & Difference & $95 \% \mathrm{Cl}$ & Tukey contrast test \\
\hline Government vs nonprofit hospitals & 1.6 & 0.8 to 2.4 & $P<0.05$ \\
\hline Government vs proprietary hospitals & 4.7 & 3.8 to 5.7 & $P<0.05$ \\
\hline Nonprofit vs proprietary hospitals & 3.1 & 2.3 to 3.9 & $P<0.05$ \\
\hline
\end{tabular}

Figure 4 Average number of patients that reported their pain as always well controlled based upon hospital ownership. Abbreviation: $\mathrm{Cl}$, confidence interval. 
of our study shows nearly a third of patients did not give high ratings when asked about pain control suggesting the guidelines may have had little change to clinical practice. Comparatively, Dahl and colleagues reported that despite statistically significant advancement in documentation of pain scale and use of nonpharmacologic interventions in addition to analgesics, there was no change in pain outcomes 12 to 18 months later. ${ }^{4}$ The fact that pain care has been the objective of many quality-improvement programs over the past decade these results suggests that major changes in the practice behaviors still need to occur.

We found a significant regional difference in pain care satisfaction with Alabama ranking highest and Hawaii ranking lowest. These differences may reflect a greater quality in pain management such as successful multimodal therapy. However, these differences may be a result of unmeasured confounders such as cultural differences in patient's expectations of pain care. The reasons for regional differences would be better understood if the HCAHPS survey included additional questions for a more comprehensive evaluation of pain care including: type of pain (acute, chronic or acute on chronic), the use of multimodal therapy and consultation from a designated pain service. Such questions will move the focus of quality improvement (QI) beyond advancing knowledge and appreciation of pain to evaluating the quality of pain care as measured by practice patterns and patient outcomes. ${ }^{20}$

Prior to public release, HCAHPS data was adjusted for confounding biases such as age and sex. However, there are certainly limitations to survey research, particularly nonresponse bias. In regards to nonresponse bias, our study must consider that $40 \%$ of US hospitals failed to provide HCAHPS data. There is often a continuum of responders and arguably those who respond early versus late may be more ardent about the context of the survey. Furthermore, nonresponders may have answered the survey much differently thus altering the pain care satisfaction rate. This however has been addressed by the developers of HCAHPS by maintaining standard survey nationwide, adjusting the data for this bias, and will be further addressed on subsequent revisions of the survey instrument.

To improve the practice of pain management, quality and standard of care must be clearly defined by the clinicians. Furthermore, interdisciplinary care plans must include patient input, use of multimodal therapy, cost-conscious, safe, and appropriate treatments. Additionally, access to specialty care must produce the necessary data to evaluate what improvement efforts actually result in improved pain outcomes. ${ }^{20}$ Medical education, from preclinical years to residency training, should include more didactics on the multidisciplinary approach to pain management so that the responsibility of providing high quality pain care is shared among all clinicians. With this common knowledge, physicians will be well equipped to evaluate and report their clinical experiences so as to promote evidence-based pain medicine. Continued advancements in both basic science and clinical pain research are crucial to improving the field of pain care. In conclusion, this study provides the evidence needed for hospitals to make pain care a priority in ultimately having patient satisfaction throughout the duration of their hospitalization. Furthermore, future research should be developed to make strategies for institutions and policy-makers to improve and optimize patient satisfaction with pain care.

\section{Disclosures}

Financial support was provided by the IASP Grant funded by the Scan/Design by Jens and Inger Bruun Foundation (RWH). The authors report no conflicts of interest in this work.

\section{References}

1. Joint Commission on Accreditation of Healthcare Organizations. Implementing the New Pain Management Standards. Oakbrook Terrace, IL: JCAHO; 2000.

2. Katz J, Jackson M, Kavanagh BP, Sandler AN: Acute pain after thoracic surgery predicts long-term post-thoractomy pain. Clin J Pain. 1996;12:50-55.

3. McGlynn EA, Asch SM, Adams J. The quality of care delivered to adults in the United States. N Engl J Med. 2003;348:2635-2645.

4. Dahl JL, Gordon D, Ward S, Skemp M, Wochos S, Schurr M. Institutionalizing pain management: The post-operative pain management quality improvement project. J Pain. 2003;4:361-371.

5. Jha AK, Li Z, Orav EJ, Epstein AM. Care in US hospitals - the Hospital Quality Alliance program. N Engl J Med. 2005;353:265-274.

6. Jha AK, Orav EJ, Zheng J, Epstein AM. Patients' perception of hospital care in the United States. N Engl J Med. 2008;359:1921-1931.

7. Fung CH, Lim YM, Mattke S, Damberg C, Shekelle PG. Systematic review: the evidence of publishing patient care performance data improves quality of care. Ann Intern Med. 2008;148:111-123.

8. Goldstein E, Farquhar M, Crofton C, Darby C, Garfinkel S. Measuring hospital care from the patients' perspective: an overview of the CAHPS Hospital Survey development process. Health Serv Res. 2005;40:1977-1995.

9. O’Malley AJ, Zaslavsky AM, Elliott MN, Zaborski L, Cleary PD. Case-mix adjustment of the CAHPS Hospital Survey. Health Serv Res 2005;40:2162-2181.

10. O’Malley AJ, Zaslavsky AM, Hays RD, Hepner KA, Keller S, Cleary PD. Exploratory factor analyses of the CAHPS Hospital Pilot Survey responses across and within medical, surgical, and obstetric services. Health Serv Res. 2005;40:2078-2095.

11. Keller S, O’Malley AJ, Hays RD, et al. Methods used to streamline the CAHPS Hospital Survey. Health Serv Res. 2005;40:2057-2077.

12. Elliott MN, Edwards C, Angeles J, Hambarsoomians K, Hays RD. Patterns of unit and item nonresponse in the CAHPS Hospital Survey. Health Serv Res. 2005;40:2096-2119.

13. de Vries H, Elliott MN, Hepner KA, Keller SD, Hays RD. Equivalence of mail and telephone responses to the CAHPS Hospital Survey. Health Serv Res. 2005;40:2120-2139. 
14. Page GG, Ben-Eliyahu S. The immune-suppressive nature of pain. Semin Oncol Nurs. 1997;13:10-15.

15. Carr DB. Preempting the memory of pain. JAMA. 1998;279:1114-1115.

16. Tsui SL, Law S, Folk M, et al. Post-operative analgesia reduces mortality and morbitidy after esophagectomy. Am J Surg. 1997;173:472-478.

17. Lee A, Chan S, Chen PP, Gin T, Lau AS. Economic evaluations of acute pain service programs: a systematic review. Clin J Pain. 2007;23: 726-733.
18. Beesdo K, Jacobi F, Hoyer J, Low NC, Höfler M, Wittchen HU. Pain associated with specific anxiety and depressive disorders in a nationally representative population sample. Soc Psychiatry Psychiatr Epidemiol. 2009 Apr 10 [Epub ahead of print].

19. Phillips DM. JCAHO pain managment standards are unveiled. JAMA. 2000;284:428-429.

20. Gordon DB, Dahl JL. Quality improvement challenges in pain management. Pain. 2004;107:1-4.

\section{Publish your work in this journal}

The Journal of Pain Research is an international, peer-reviewed, open access, online journal that welcomes laboratory and clinical findings in the fields of pain research and the prevention and management of pain. Original research, reviews, symposium reports, hypothesis formation and commentaries are all considered for publication.

\section{Dovepress}

The manuscript management system is completely online and includes a very quick and fair peer-review system, which is all easy to use. Visit http://www.dovepress.com/testimonials.php to read real quotes from published authors. 